\title{
Quasinormal modes and classical wave propagation in analogue black holes
}

\author{
Emanuele Bert* \\ McDonnell Center for the Space Sciences, Department of Physics, \\ Washington University, St. Louis, Missouri 63130, USA \\ Vitor Cardosd $\AA^{\dagger}$ \\ Centro de Física Computacional, Universidade de Coimbra, P-3004-516 Coimbra, Portugal \\ José P. S. Lemơ⿱ \\ Centro Multidisciplinar de Astrofísica - CENTRA, Departamento de Física, \\ Instituto Superior Técnico, Av. Rovisco Pais 1, 1049-001 Lisboa, Portugal
}

(Dated: August 20, 2018)

\begin{abstract}
Many properties of black holes can be studied using acoustic analogues in the laboratory through the propagation of sound waves. We investigate in detail sound wave propagation in a rotating acoustic $(2+1)$-dimensional black hole, which corresponds to the "draining bathtub" fluid flow. We compute the quasinormal mode frequencies of this system and discuss late-time power-law tails. Due to the presence of an ergoregion, waves in a rotating acoustic black hole can be superradiantly amplified. We compute superradiant reflection coefficients and instability timescales for the acoustic black hole bomb, the equivalent of the Press-Teukolsky black hole bomb. Finally we discuss quasinormal modes and late-time tails in a non-rotating canonical acoustic black hole, corresponding to an incompressible, spherically symmetric $(3+1)$-dimensional fluid flow.
\end{abstract}

PACS numbers: 04.70.-s, 43.20.+g, 04.80.Cc

\section{INTRODUCTION}

Black holes are one of the most fascinating predictions of general relativity. Being a solution of the classical Einstein field equations in vacuum, they are the simplest objects which can be built out of spacetime itself. Hawking [1] showed that when quantum effects are taken into account, black holes are not really black: they slowly evaporate by emitting an almost thermal radiation. Hawking's prediction has been tested time and again in very different ways. It is now clear that the appearance of Hawking radiation does not depend on the dynamics of the Einstein equations, but only on their kinematical structure, and more specifically on the existence of an apparent horizon [2, 3]. The discovery of Hawking radiation uncovered a number of fundamental questions: among them the information puzzle, the issue of the black hole final state, and so on. For these reasons an experimental verification of the Hawking effect would be of the utmost importance. Unfortunately astrophysical black holes, having a temperature much smaller than the temperature of the cosmic microwave background, accrete matter more efficiently than they evaporate. An alternative could be provided by the existence of large extra dimensions: if gravity is effective at the TeV scale, black holes could be produced in particle accelerators [4]. However, even if these mini-black holes are actually produced in colliders, it is unlikely that they will yield any conclusive evidence of the existence of Hawking radiation.

Prospects for detecting Hawking radiation changed when Unruh [5] realized that the basic ingredients of Hawking radiation can experimentally be reproduced in the laboratory. Since Hawking radiation crucially depends on the existence of an apparent horizon, the experimental setup should display the essential features that define apparent horizons in general relativity. Unruh considered precisely such a system: a fluid moving with a space-dependent velocity, for example water flowing through a nozzle. Where the fluid velocity exceeds the sound velocity we get the equivalent of an apparent horizon for sound waves. This is the acoustic analogue of a black hole, or a dumb hole. Following on Unruh's dumb hole proposal many different kinds of analogue black holes have been devised, based on condensed matter physics, slow light etcetera 6, 7]. At present the Hawking temperatures associated to these analogues are too low to be detectable, but the situation is likely to change in the near future 8,9$]$.

In order to detect Hawking radiation, a full understanding of the classical physics of analogue black holes is

\footnotetext{
*Electronic address: berti@iap.fr

${ }^{\dagger}$ Electronic address: vcardoso@teor.fis.uc.pt

${ }^{\ddagger}$ Electronic address: lemos@fisica.ist.utl.pt
} 
necessary. Not only must one control what happens in the experimental situation, but the understanding of classical phenomena may bring clues on how to favor the probabilities to detect Hawking radiation. It is also worth stressing that some purely classical phenomena shed light on quantum aspects of (analogue and general-relativistic) black hole physics. For example, positive and negative norm mixing at the horizon leads to non-trivial Bogoliubov coefficients in the calculations of Hawking radiation 10]; superradiant instabilities of the Kerr metric are related to the quantum process of Schwinger pair production 11]; and more speculatively (classical) highly damped black hole oscillations could be related to area quantization [12].

In this paper we carry out a comprehensive study of wave propagation in analogue black holes. We consider in detail two acoustic black hole metrics: the "draining bathtub" model for a $(2+1)$-dimensional fluid flow and the "canonical" metric for a non-rotating, spherically symmetric $(3+1)$-dimensional acoustic black hole 7 ]. For each acoustic metric we compute the characteristic oscillation frequencies using a WKB approach. These complex characteristic frequencies, called quasinormal modes (QNMs), play a very important role in the classical physics of black holes. They govern the late time behavior of waves propagating outside the black hole: any perturbation of the black hole, after an initial transient, will damp exponentially in the so-called "ringdown phase". The frequencies and damping times of this ringdown signal depend only on the black hole parameters, such as the mass, charge and angular momentum 13], and can therefore be used to estimate black hole parameters from observational data [14]. Therefore, in cases where a "formal" definition of (say) the mass and angular momentum of acoustic black holes is missing, quasinormal (QN) frequencies may be used to define these quantities operationally. According to recent speculations, highly damped QNMs may yield some information on quantum properties of a black hole. In particular, it has been conjectured that highly damped QN frequencies could be linked to black hole area quantization 12. Motivated by these conjectures we also study highly damped QNMs of acoustic black holes. We find that for a $(2+1)$-dimensional acoustic black hole there are no asymptotic QN frequencies, whereas for the $(3+1)$-dimensional canonical black hole QN frequencies are asymptotically given by $4 \pi \omega=\log [(3-\sqrt{5}) / 2]-\mathrm{i}(2 n+1) \pi$. This result does not seem to support any of the recent conjectures, but perhaps this is no surprise, since Hod's argument relies heavily on black hole thermodynamics. Even the very formulation of the laws of black hole thermodynamics for analogue black holes is a non-trivial matter [2].

After the exponential decay characteristic of the ringdown phase, black hole perturbations decay with a power-law tail [15] due to backscattering off the background curvature. Here we compute the late-time tails of wave propagation in the draining bathtub and canonical acoustic black hole metrics. We show that for the $(2+1)$-dimensional fluid flow the field falloff at very late times is of the form $\Psi \sim t^{-(2 m+1)}$, where $m$ is the angular quantum number. This time exponent is characteristic of any $(2+1)$-dimensional flow, and not just of a black hole. For the $(3+1)$-dimensional canonical acoustic geometry, the field falloff is of the form $\Psi \sim t^{-(2 l+6)}$.

The rotating draining bathtub metric, possessing an ergoregion, can display the phenomenon of superradiance [16, 17, 18]. We compute reflection coefficients for this superradiant scattering by numerical integration of the relevant equations. Enclosing the acoustic black hole by a reflecting mirror we can exploit superradiance to destabilize the system, making an initial perturbation grow exponentially with time: we have an "acoustic black hole bomb" [19, 20, 21]. We compute analytically and numerically the frequencies and growing timescales for this instability. An interesting feature of acoustic geometries is that the acoustic black hole spin can be varied independently of the black hole mass. Therefore, at variance with the Kerr metric, the spin can be made (at least in principle) very large, and rotational superradiance in acoustic black holes can be very efficient. It should not be difficult to set up an experimental apparatus to observe an acoustic black hole bomb in the lab. A concrete example of an experimental setup in which this idea can be realized with moderate experimental effort has been fully worked out by Schützhold and Unruh [16], and is based on the study of gravity waves in shallow water.

The paper is organized as follows. In Section III we discuss the $(2+1)$-dimensional draining bathtub metric. We first introduce the formalism describing sound propagation in this acoustic metric, and shortly describe the possible experimental setup (gravity waves in a shallow basin). Then we compute QNMs, discuss late-time tails, introduce the phenomenon of superradiant amplification and quantify the timescales for the acoustic black hole bomb instability. In Section III we repeat the analysis for the canonical $(3+1)$-dimensional acoustic black hole metric (of course, the absence of an ergoregion means that we have no superradiance in this case). The conclusions follow in Section IV]

\section{DRAINING BATHTUB: A ROTATING ACOUSTIC BLACK HOLE}

In this Section we consider a simple "draining bathtub" model, first introduced in 7], for a rotating acoustic black hole. We write down the acoustic metric and the wave equation describing sound propagation in this model, and describe a concrete example for a possible experimental setup. We compute QNMs and discuss the late-time tail behavior of the system. Due to the presence of an ergoregion, sound waves in this acoustic black hole can be superradiantly amplified. We quantify this amplification and discuss the possibility to build an acoustic black hole bomb in the lab. 


\section{A. Formalism and basic equations}

Consider a fluid having (background) density $\rho$. Assume the fluid to be locally irrotational (vorticity free), barotropic and inviscid. From the equation of continuity, the radial component of the fluid velocity satisfies $\rho v^{r} \sim 1 / r$. Irrotationality implies that the tangential component of the velocity satisfies $v^{\theta} \sim 1 / r$. By conservation of angular momentum we have $\rho v^{\theta} \sim 1 / r$, so that the background density of the fluid $\rho$ is constant. In turn, this means that the background pressure $p$ and the speed of sound $c$ are constants. The acoustic metric describing the propagation of sound waves in this "draining bathtub" fluid flow is 7]:

$$
d s^{2}=-\left(c^{2}-\frac{A^{2}+B^{2}}{r^{2}}\right) d t^{2}+\frac{2 A}{r} d r d t-2 B d \phi d t+d r^{2}+r^{2} d \phi^{2}
$$

Here $A$ and $B$ are arbitrary real positive constants related to the radial and angular components of the background fluid velocity:

$$
\vec{v}=\frac{-A \hat{r}+B \hat{\theta}}{r}
$$

This flow velocity can be obtained as the gradient of a velocity potential, $\vec{v}=\nabla \psi$, where

$$
\psi=-A \log (r / a)+B \phi
$$

and $a$ is some (irrelevant) length scale.

In the non-rotating limit $B=0$ the metric (11) reduces to a standard Painlevé-Gullstrand-Lemaitre type metric 22. The acoustic event horizon is located at $r_{H}=A / c$, and the ergosphere forms at $r_{E S}=\left(A^{2}+B^{2}\right)^{1 / 2} / c$. Unruh 5] first realized that the propagation of a sound wave in a barotropic inviscid fluid with irrotational flow is described by the Klein-Gordon equation $\nabla_{\mu} \nabla^{\mu} \Psi=0$ for a massless field $\Psi$ in a Lorentzian acoustic geometry, which in our case takes the form (1). In our acoustic geometry we can separate variables by the substitution

$$
\Psi(t, r, \phi)=R(r) e^{\mathrm{i}(m \phi-\omega t)} .
$$

Then we obtain a simple ordinary differential equation for the radial variable:

$$
R_{, r r}+P_{1}(r) R_{, r}+Q_{1}(r) R=0
$$

where

$$
\begin{aligned}
P_{1}(r) & =\frac{A^{2}+r^{2} c^{2}+2 \mathrm{i} A\left(B m-r^{2} \omega\right)}{r\left(r^{2} c^{2}-A^{2}\right)} \\
Q_{1}(r) & =-\frac{2 \mathrm{i} A B m-B^{2} m^{2}+c^{2} m^{2} r^{2}+2 B m \omega r^{2}-r^{4} \omega^{2}}{r^{2}\left(r^{2} c^{2}-A^{2}\right)} .
\end{aligned}
$$

Notice that if we take the incompressible fluid limit $c \rightarrow \infty$ we get well known equations from fluid dynamics 23, 24]. We now introduce a tortoise coordinate $r_{*}$ defined by the condition

$$
\frac{d r_{*}}{d r}=\Delta
$$

where $\Delta \equiv\left(1-A^{2} / c^{2} r^{2}\right)^{-1}$. Explicitly,

$$
r_{*}=r+\frac{A}{2 c} \log \left|\frac{c r-A}{c r+A}\right| .
$$

Setting $R=Z H$ we get:

$$
Z \Delta^{2} H_{, r_{*} r_{*}}+\left[\Delta\left(2 Z_{, r}+P_{1} Z\right)+\Delta^{\prime} Z\right] H_{, r_{*}}+\left(Z_{, r r}+P_{1} Z_{, r}+Q_{1} Z\right) H=0 .
$$

To obtain a Schrödinger-like equation we impose the coefficient of $H_{, r_{*}}$ to be zero:

$$
Z_{, r}+\frac{1}{2}\left[\frac{\left(c^{2} r^{2}-A^{2}\right)+2 \mathrm{i} A\left(B m-r^{2} \omega\right)}{r\left(c^{2} r^{2}-A^{2}\right)}\right] Z=0
$$


A solution of this equation can easily be found:

$$
Z=r^{1 / 2} \exp \left[\left(\frac{\mathrm{i} B m}{A}-1\right) \ln r+\frac{\mathrm{i}\left(A^{2} \omega-B m c^{2}\right)}{2 A c^{2}} \ln \left(c^{2} r^{2}-A^{2}\right)\right] .
$$

Replacing this solution in Eq. (9) we finally get the wave equation:

$$
H_{, r_{*} r_{*}}+\left\{\frac{1}{c^{2}}\left(\omega-\frac{B m}{r^{2}}\right)^{2}-\left(\frac{c^{2} r^{2}-A^{2}}{c^{2} r^{2}}\right)\left[\frac{1}{r^{2}}\left(m^{2}-\frac{1}{4}\right)+\frac{5 A^{2}}{4 r^{4} c^{2}}\right]\right\} H=0 .
$$

The derivation given here is very similar to the one in [17], but we have corrected some typos in that paper. In particular notice that the potential is the same as equation (10) in [17], except for a factor $1 / 4$ in the last term. Some physical properties of our "draining bathtub" metric are more apparent if we cast the metric in a Kerr-like form performing the following coordinate transformation (where again we correct some typos in [18]):

$$
d t \rightarrow d \tilde{t}+\frac{A r}{r^{2} c^{2}-A^{2}} d r, \quad d \phi \rightarrow d \tilde{\phi}+\frac{B A}{r\left(r^{2} c^{2}-A^{2}\right)} d r .
$$

Then the effective metric takes the form

$$
d s^{2}=-\left(1-\frac{A^{2}+B^{2}}{c^{2} r^{2}}\right) c^{2} d \tilde{t}^{2}+\left(1-\frac{A^{2}}{c^{2} r^{2}}\right)^{-1} d r^{2}-2 B d \tilde{\phi} d \tilde{t}+r^{2} d \tilde{\phi}^{2} .
$$

Notice an important difference between this acoustic metric and the Kerr metric: in the $(t, t)$ component of the metric (14) the parameters $A$ and $B$ appear as a sum of squares. This means that, at least in principle, there is no upper bound for the rotational parameter $B$ in the acoustic black hole metric, contrary to what happens in the Kerr geometry. Separating variables by the substitution

$$
\Psi(\tilde{t}, r, \tilde{\phi})=\sqrt{r} H(r) e^{\mathrm{i}(m \tilde{\phi}-\omega \tilde{t})},
$$

one can show that the radial function $H(r)$ is again a solution of equation (12).

The wave equation (12) can be recast in a more convenient form by the following rescaling: $\hat{r}=r A / c, \hat{\omega}=\omega A / c^{2}$, $\hat{B}=B / A$. We get

$$
H_{, \hat{r}_{*} \hat{r}_{*}}+Q H=0,
$$

where the generalized potential

$$
Q \equiv\left\{\left(\hat{\omega}-\frac{\hat{B} m}{\hat{r}^{2}}\right)^{2}-V\right\}, \quad V \equiv\left(\frac{\hat{r}^{2}-1}{\hat{r}^{2}}\right)\left[\frac{1}{\hat{r}^{2}}\left(m^{2}-\frac{1}{4}\right)+\frac{5}{4 \hat{r}^{4}}\right] .
$$

The rescaling effectively sets $A=c=1$ in the original wave equation, and picks units such that the acoustic horizon $\hat{r}_{H}=1$. From now on we shall omit hats in all quantities (unless otherwise stated). The rescaled wave equation (16) will be the starting point of our analysis of QNMs, late-time tails and superradiant phenomena. Before giving details of this analysis we describe a possible experimental setup in which all of the classical physics we are going to discuss could, at least in principle, be reproduced.

\section{B. A possible experimental setup}

The acoustic metric (11) can be realized in a simple experimental setup, that was described in detail by Schützhold and Unruh [16]. In this setup it no longer describes a sonic analogue but rather a shallow basin gravity wave analogue of a black hole. The idea is to use gravity waves in a viscosity free, incompressible liquid with irrotational flow: under appropriate circumstances, one can envisage the use of common fluids like water or mercury. Schützhold and Unruh assumed a shallow water, long wavelength approximation: the gravity wave amplitude $\delta h$, their wavelength $\lambda$ and the depth of the basin $h_{B}$ are such that $\delta h \ll h_{B} \ll \lambda$. Relaxing the assumptions that the bottom of the tank and the background flow surfaces are flat and parallel, they showed that the most general rotationally symmetric and locally irrotational background flow profile can be described precisely by the draining bathtub metric (11) when the radial slope of the bottom of the tank - that in cylindrical coordinates $(z, r, \phi)$ will be described by some function $f(r)$ - 
is small: $f^{\prime}(r) \ll 1$. In this gravity wave black hole analogue the constants $A$ and $B$ are proportional to the radial and tangential components of the background flow velocity:

$$
v^{\phi}=\frac{B}{r^{2}}, \quad v^{r}=-\frac{A h_{\infty}}{r h \sqrt{1+f^{\prime}(r)^{2}}},
$$

where $h_{\infty}$ is the height of the tank far from the black hole, and the slope of the tank satisfies the relation

$$
f(r)=-\frac{\left(A^{2}+B^{2}\right)}{g r^{2}} .
$$

In the previous equations $g$ is the gravitational acceleration, related to the constant $c$ of the acoustic black hole metric (11) by the relation

$$
c=\sqrt{g h_{\infty}} .
$$

One of the main advantages of this acoustic black hole is apparent from this equation: the speed of the gravity waves can simply be tuned to one's needs by adjusting the height of the basin $h_{\infty}$. Another advantage, that we will not exploit here, is that the inclusion of surface tension and viscosity can be used to manipulate the waves' dispersion relation. To be concrete we will sometimes consider the following plausible choice of physical parameters, as suggested in [16]: gravity waves of amplitude $\delta h \sim 1 \mathrm{~mm}$ and wavelength $\lambda \sim 10 \mathrm{~cm}$; a tank of height $h_{\infty} \sim 1 \mathrm{~cm}$ (so that $c \sim 0.31 \mathrm{~m} \mathrm{~s}^{-1}$ ); and a typical characteristic size of the acoustic black hole horizon $r_{H} \sim 1 \mathrm{~m}$, corresponding to $A=c r_{H} \sim 0.31 \mathrm{~m}^{2} \mathrm{~s}^{-1}$.

\section{Quasinormal modes}

Numerical and analytical studies of a fairly general class of initial data show that the evolution of the perturbations of a black hole spacetime can roughly be divided in three parts: (i) The first part is the prompt response at very early times. In this phase, which is the obvious counterpart of light cone propagation, the form of the signal depends strongly on the initial conditions. (ii) At intermediate times the signal is dominated by an exponential decay, whose frequencies and damping times are determined by the black hole QNMs. In this "ringdown" phase the signal depends entirely on the black hole parameters (typically mass, charge and angular momentum). (iii) Due to backscattering off the spacetime curvature, at late times the propagating wave leaves a "tail" behind, usually a power law falloff of the field. This power law seems to be highly independent of the initial data, and persists even if there is no black hole horizon. In fact it only depends on the asymptotic far region. In the following we study the QNM ringing phase of our acoustic black hole metric; then we will consider its late-time behavior.

The characteristic QNMs of the rotating acoustic black hole can be defined in the usual way, imposing appropriate boundary conditions and solving the corresponding eigenvalue problem. Close to the event horizon the solutions of equation (16) behave as

$$
H \sim e^{ \pm \mathrm{i}(\omega-B m) r_{*}} .
$$

Classically, only ingoing waves - that is, waves falling into the black hole - should be present at the horizon. This means (according to our conventions on the time dependence of the perturbations) that we must choose the minus sign in the exponential. At spatial infinity the solutions of (16) behave as

$$
H \sim e^{ \pm \mathrm{i} \omega r_{*}} .
$$

In this case we require that only outgoing waves (waves leaving the domain under study) should be present, and correspondingly choose the plus sign in the exponential. This boundary condition at infinity may be cause for objections. Indeed, no actual physical apparatus will be accurately described by these boundary conditions: a real acoustic black hole experiment will certainly not extend out to infinity. However, we may imagine using some absorbing device to simulate the "purely outgoing" wave conditions at infinity (for another example in which an absorbing device modeling spatial infinity could be required, cf. Section XI of [16] - in particular their Fig. 5). In any event, later on we shall consider the alternative possibility of "boxed" boundary conditions, describing a closed system. Boxed boundary conditions were also considered in [20].

For assigned values of the rotational parameter $B$ and of the angular index $m$ there is a discrete (and infinite) set of QN frequencies, $\omega_{Q N}$, satisfying the wave equation (16) with boundary conditions specified by Eqs. (21) and (22). The QN frequencies are in general complex numbers, the imaginary part describing the decay or growth of 
TABLE I: The fundamental $(n=0) \mathrm{QN}$ frequencies for the non-rotating acoustic black hole, using three WKB computational schemes. $\omega_{Q N}^{(1)}$ is the result for the QN frequency using only the lowest approximation [25], $\omega_{Q N}^{(3)}$ is the value obtained using 3rd order improvements [26], and finally $\omega_{Q N}^{(6)}$ was computed using 6th order corrections [28]. Notice how for $m>1$ the three schemes yield very similar answers.

\begin{tabular}{c|c|c|c}
\hline \hline$m$ & $\omega_{Q N}^{(1)}$ & $\omega_{Q N}^{(3)}$ & $\omega_{Q N}^{(6)}$ \\
\hline \hline 1 & $0.696-0.353 \mathrm{i}$ & $0.321-0.389 \mathrm{i}$ & $0.427-0.330 \mathrm{i}$ \\
2 & $1.105-0.349 \mathrm{i}$ & $0.940-0.353 \mathrm{i}$ & $0.945-0.344 \mathrm{i}$ \\
3 & $1.571-0.351 \mathrm{i}$ & $1.465-0.353 \mathrm{i}$ & $1.468-0.352 \mathrm{i}$ \\
4 & $2.054-0.352 \mathrm{i}$ & $1.975-0.353 \mathrm{i}$ & $1.976-0.353 \mathrm{i}$ \\
\hline \hline
\end{tabular}

the perturbation, because the time dependence is given by $e^{-\mathrm{i} \omega t}$. We expect the black hole to be stable against small perturbations, and therefore $\omega_{Q N}$ is expected to have a negative imaginary part, so that the perturbation decays exponentially as time goes by. We could not prove stability of the draining bathtub metric in general, but we managed to derive upper bounds on the frequencies of unstable modes (if they exist at all). We give the derivation of these upper bounds in Appendix As usual, we will order the QN frequencies $\omega_{Q N}$ according to the absolute value of their imaginary part: the fundamental mode (labeled by an integer $n=0$ ) will have the smallest imaginary part (in modulus), and so on.

\section{Slowly decaying modes of non-rotating black holes}

The lowest QNMs control the ringing behavior of any classical perturbation outside the black hole. Higher overtones, having a larger imaginary part, are damped more quickly and play a negligible role. In particular, it is known 13. that the fundamental $(n=0)$ QNM effectively determines the response of the black hole to exterior perturbations. For these slowly damped QNMs, a WKB approximation - as developed by Schutz, Will and others [25, 26, 27] - is accurate enough. As a convergence check, we will sometimes extend the WKB treatment to sixth order 28]. The WKB method demands that the generalized potential $Q$ defined by Eq. (17) has a single maximum outside the horizon. For non-rotating black holes $(B=0)$ this is certainly true provided $m$ is not zero, and one can easily extract the lowest QN frequencies in this case. We show them in Table \ where we also show the convergence of the WKB scheme as the order of approximation increases. For $m=0$ the situation is not so simple, and we haven't been able to extract the QN frequencies using this method, because the potential possesses two extrema. Due to the symmetry of the potential (17), QN frequencies of non-rotating black holes are independent of the sign of $m$ (to any positive QN frequency for positive $m$ corresponds a negative QN frequency for negative $m$ ).

From Table \we see that the imaginary part is nearly constant as a function of $m$, whereas the real part approximately scales with $m$. Indeed, in the limit of large $m$ one can show directly from the WKB formula 25] that $\omega_{Q N}$ behaves as

$$
\omega \sim \frac{m}{2}-i \frac{2 n+1}{2 \sqrt{2}}, m \rightarrow \infty,
$$

and indeed already for $m=4$ this formula yields very good agreement with the results shown in Table \ This very same result can also be obtained using a Pöschl-Teller fitting potential [29]. Just to give an idea of the orders of magnitude involved consider an $m=2$ mode: if we take the "typical" gravity wave analogue experimental parameters of Section $\amalg B$ we get (for the fundamental QNM with $m=2$ ) a frequency $\omega_{R}=0.945 \times c^{2} / A=0.293 \mathrm{~Hz}$ and a damping timescale $\tau=1 /\left|\omega_{I}\right|=1 / 0.344 \times A / c^{2}=9.37 \mathrm{~s}$.

\section{Slowly decaying modes of rotating black holes}

For rotating black holes, the generalized potential has more than one extremum. This immediately raises problems for the applicability of the WKB technique. Previous work on the Kerr geometry [26, 27] showed that the WKB method can still be used, yielding good results, as long as the rotation parameter is small. The way to handle the several extrema of the potential is the following: start with $B=0$ and compute the roots of $d Q / d r_{*}=0$ (to find the extrema; as we said before, for $B=0$ the generalized potential $Q$ has a single maximum outside the horizon as long as $m \neq 0)$. Compute $\omega_{Q N}$ in the non-rotating case. Then add some small rotation $B$ to the black hole, and compute 

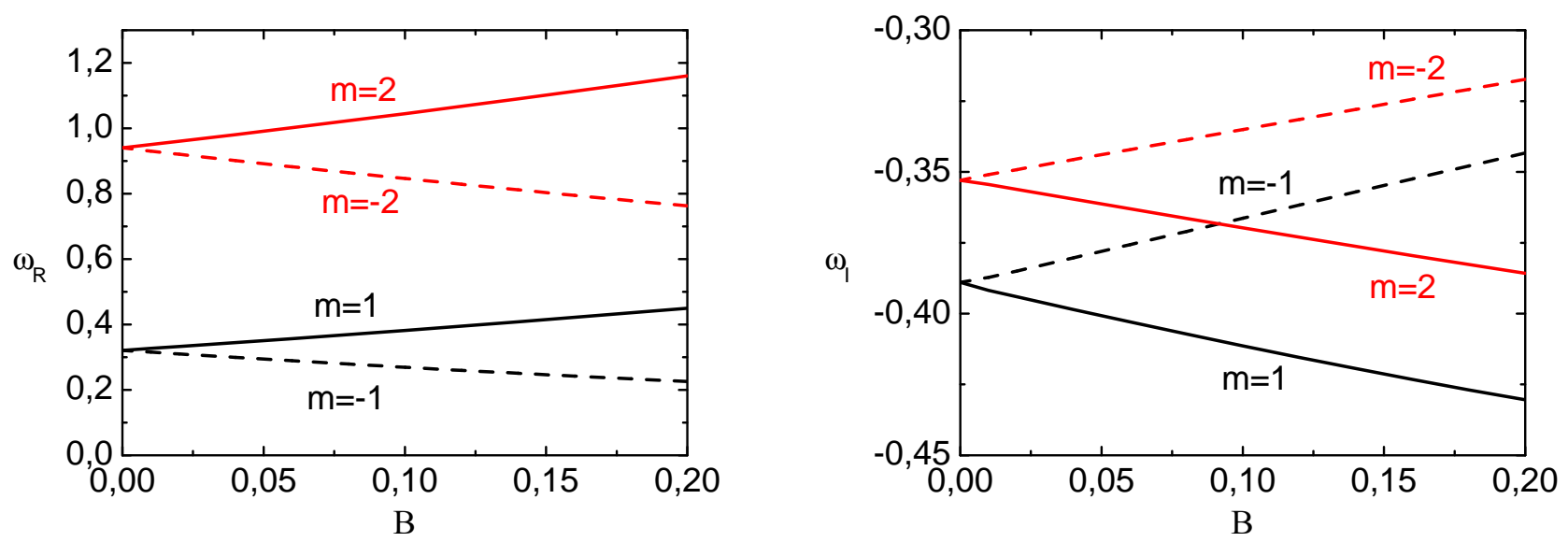

FIG. 1: In the left panel we show the real part of the fundamental QN frequency $\omega_{R}$ (and in the right panel we show the imaginary part $\left.\omega_{I}\right)$ as a function of the rotation parameter $B$, for selected values of $m$. For $m>0(m<0) \omega_{R}$ and $\left|\omega_{I}\right|$ increase (decrease) with rotation.

the roots of $d Q / d r_{*}=0$. Now the roots will depend on $\omega$, but only one root yields the correct non-rotating limit as $B \rightarrow 0$. It is this root that corresponds to the QNMs of the rotating black hole. Our results, which can be trusted for $B \lesssim 0.2$, are shown in Fig. [1 For $m>0(m<0) \omega_{R}$ and $\left|\omega_{I}\right|$ increase (decrease) with rotation, at least in the range in which the WKB method can be applied. The QN frequency change with rotation is not dramatic, but it can probably be used to apply a "fingerprint analysis" of the acoustic black hole parameters á la Echeverria [14]: that is, once we measure at least two QNM frequencies we may infer the acoustic black hole parameters $A$ and $B$. Carrying out such experiments in the lab can shed some light on the applicability of similar ideas to test the no-hair theorem in the astrophysical context [30]. A more complete analysis of the QNMs is still needed to probe the high rotation regime: the results shown in Fig. 1 seem to indicate that instability may set in for large $\mathrm{Bm}$. A continued fraction analysis could be used to test this hypothesis, but it is beyond the scopes of this paper [31].

\section{Highly damped modes}

QNMs with a large imaginary part, i.e. with a very large overtone number $n$, have recently become a subject of intense scrutiny for black holes in general relativity and similar theories. The interest in these modes comes from Hod's proposal 12 that they could be related to black hole area quantization, and from Dreyer's suggestion that a similar argument could be used to fix the Barbero-Immirzi parameter in Loop Quantum Gravity [32]. In this context, an analytical calculation of highly damped QNMs was first carried out by Motl for the Schwarzschild black hole 33]. Subsequently Motl and Neitzke 34] used a complex-integration technique to compute highly-damped QNMs of the Schwarzschild and Reissner-Nordström black holes. Their analytical results are in striking agreement with numerical data [35, 36] and alternative analytical calculations 37]. The complex integration method has also been generalized with success to other black hole geometries [38, 39], yielding again predictions in excellent agreement with the numerical results [40]. In view of this, we shall now build on the results and techniques of [34] to compute highly damped QNMs of a rotating acoustic black hole.

Let us first consider a non-rotating black hole. In the limit $B \rightarrow 0$ the wave equation (16) reduces to

$$
\frac{d^{2} H}{d r_{*}^{2}}+\left[\omega^{2}-V(r)\right] H=0
$$

where the potential

$$
V(r) \equiv\left(1-\frac{1}{r^{2}}\right)\left(\frac{m^{2}-1 / 4}{r^{2}}+\frac{5}{4 r^{4}}\right)
$$

Following $(\underline{34}])$, we can determine the highly damped QNMs looking at the behaviour of the potential near the 
singular point $r=0$. In our case the leading term as $r \rightarrow 0$ is

$$
V \sim-\frac{5}{4 r^{6}},
$$

and in the same limit we have $r_{*} \sim-r^{3} / 3$. Thus

$$
V \sim-\frac{5}{36 r_{*}^{2}}=\frac{j^{2}-1}{4 r_{*}^{2}},
$$

for $j=2 / 3$ (this is precisely the same power-law behavior found in the case of the Schwarzschild black hole, except for the different value of $j$ ). The result in [34] carries over directly:

$$
e^{4 \pi \omega}=-(1+2 \cos \pi j) .
$$

However now $(1+2 \cos \pi j)=0$. This means that there are no asymptotic QN frequencies for this black hole. A similar situation occurs for electromagnetic perturbations of a five-dimensional black hole [40]. If we add rotation to the black hole, i.e., if $B$ is non-zero, a similar analysis [41] implies that the asymptotic QN frequencies behave as

$$
e^{4 \pi(\omega-m B)}=-(1+2 \cos \pi j),
$$

where again $j=2 / 3$. Thus also in this case there will be no asymptotic QN frequencies.

This result is quite puzzling. It could mean either that the real part grows without bound, or that it just doesn't converge to any finite value. In any case, an application of Hod's conjecture to these black holes seems impossible: the very definition of the classical laws of black hole thermodynamics is non-trivial in the analogue case. In fact, as argued by Visser [2], the laws of black hole thermodynamics arise solely from the Einstein equations for the metric; from a different perspective, it has been shown that the Einstein equations themselves can be derived from purely thermodynamical arguments 42]. A well-known "weakness" of analogue models is the fact that they can be used to reproduce the kinematical aspects of the Einstein equations, but not their dynamical aspects (see eg. [43] for the implications of this distinction on the causal structure of acoustic spacetimes). Hod's arguments [12] assume a thermodynamic relation between black hole surface area and entropy: even if Hod's conjecture is true, the absence of any such relation for analogues could explain the missing link between the QNM spectrum and area quantization.

\section{Late-time tails}

After the exponential QNM decay characteristic of the ringdown phase black hole perturbations usually decay with a power-law tail [15], due to the backscattering of waves off the background curvature. The existence of late-time tails in black hole spacetimes is by now well established. A strong body of evidence comes from analytical and numerical calculations using linear perturbation theory and even non-linear evolutions, for massless or massive fields 15, 44, 45, 46, 47]. In a very complete analysis, Ching, Leung, Suen and Young [45, 46] considered the late-time tails appearing when one deals with evolution equations of the form (16), and the potential $V$ is of the form

$$
V\left(r_{*}\right) \sim \frac{\nu(\nu+1)}{r_{*}^{2}}+\frac{c_{1} \log r_{*}+c_{2}}{r_{*}^{\alpha}}, \quad r_{*} \rightarrow \infty .
$$

By a careful study of the branch cut contribution to the associated Green's function they concluded that in general the late-time behavior is dictated by a power-law or by a power-law times a logarithm. The exponents of the power-law depend on the leading term at very large spatial distances. The case of interest for us here is when $c_{1}=0$. Their conclusions, which we will therefore restrict to the $c_{1}=0$ case, are (see Table 1 in [45] or [46]):

(i) if $\nu$ is an integer the term $\nu(\nu+1) / r_{*}^{2}$ does not contribute to the late-time tail. Since this term represents just the pure centrifugal barrier, characteristic of flat space, one can expect that indeed it does not contribute, at least in four-dimensional spacetimes. Therefore, for integer $\nu$, it is the $c_{2} / r_{*}^{\alpha}$ term that contributes to the late-time tail. In this case the authors of [45, 46] find that the tail is given by a power-law,

$$
\Psi \sim t^{-\mu}, \mu>2 \nu+\alpha, \alpha \text { odd integer }<2 \nu+3 .
$$

where the exponent $\mu=2 \nu+2 \alpha-2$. For all other real $\alpha$, the tail is

$$
\Psi \sim t^{-(2 \nu+\alpha)} \text {, all other real } \alpha .
$$


(ii) if $\nu$ is not an integer, then the main contribution to the late-time tail comes from the $\nu(\nu+1) / r_{*}^{2}$ term. In this case the tail is

$$
\Psi \sim t^{-(2 \nu+2)}, \text { non integer } \nu .
$$

As an example of non-integer $\nu$, Cardoso et al. 47] have shown that the late-time tails of wave propagation appearing in odd dimensional spacetimes do not depend on the presence of the black hole at all. For odd dimensions the powerlaw is determined not by the presence of the black hole, but by the very fact that the spacetime is odd dimensional. In this case the field decays as

$$
\Psi \sim t^{-(2 l+D-2)}
$$

where $l$ is the angular index determining the angular dependence of the field, and $D$ the number of spacetime dimensions. One can show directly from the flat space Green's function that such a power-law is indeed expected in flat, odd dimensional spacetimes.

From the aforementioned arguments we should expect late-time tails in our draining bathtub acoustic black hole to be directly related to the dimensionality of the underlying flow, and not to the presence of a black hole, since we are dealing with a $(2+1)$-dimensional flow. Indeed, at leading order the potential behaves as

$$
V \sim \frac{m^{2}-1 / 4}{r_{*}^{2}}=\frac{\nu(\nu+1)}{r_{*}^{2}},
$$

where $\nu=m-1 / 2$. We are thus in case (ii) above, because $\nu$ is not an integer. So any perturbation in the vicinities of this black hole will die out as a late-time tail of the form

$$
H \sim t^{-(2 m+1)} .
$$

This power-law is just the one given by the general expression (34), if one substitutes $D=3$.

Strictly speaking, the asymptotic form (35) for the potential is valid only for non-rotating acoustic black holes. For the general rotating case the leading term in the potential is $\omega$-dependent:

$$
V \sim \frac{m^{2}-1 / 4}{r_{*}^{2}}=\frac{\nu(\nu+1)+2 \omega B m}{r_{*}^{2}} .
$$

Since late-times are associated with small frequencies, and the term $\nu(\nu+1)$ gives a contribution to the late-time tail, then it follows that the late-time tails of rotating acoustic black holes are the same as those for non-rotating black holes, expression (36).

\section{E. Superradiance}

Superradiance is a general phenomenon in physics. Inertial motion superradiance has long been known [48], and refers to the possibility that a (possibly electrically neutral) object endowed with internal structure, moving uniformly through a medium, may emit photons even when it starts off in its ground state. Some examples of inertial motion superradiance include the Cherenkov effect, the Landau criterion for disappearance of superfluidity, and Mach shocks for solid objects travelling through a fluid (cf. [49] for a discussion). Non-inertial rotational motion also produces superradiance. This was discovered by Zel'dovich [50], who pointed out that a cylinder made of absorbing material and rotating around its axis with frequency $\Omega$ can amplify modes of scalar or electromagnetic radiation of frequency $\omega$, provided the condition

$$
\omega<m \Omega
$$

(where $m$ is the azimuthal quantum number with respect to the axis of rotation) is satisfied. Zel'dovich realized that, accounting for quantum effects, the rotating object should emit spontaneously in this superradiant regime. He then suggested that a Kerr black hole whose angular velocity at the horizon is $\Omega$ will show both amplification and spontaneous emission when the condition (38) for superradiance is satisfied. This suggestion was put on firmer ground by a substantial body of work [51]. In particular, it became clear that (even at the purely classical level) superradiance is required to satisfy Hawking's area theorem [19, 52].

Superradiance is essentially related to the presence of an ergosphere, allowing the extraction of rotational energy from a black hole through a wave equivalent of the Penrose process [53]. Under certain conditions, superradiance can 
be used to induce instabilities in Kerr black holes 11. Indeed, all spacetimes admitting an ergosphere and no horizon are unstable due to rotational superradiance. This was shown rigorously in [54], but the growth rate of the instability is too slow to observe it in an astrophysical context 55 . Kerr black holes are stable, but if enclosed by a reflecting mirror they can become unstable due to superradiance [20, 56]; we will discuss this "black hole bomb" instability as applied to the analogue acoustic black hole.

The possibility to observe rotational superradiance in analogue black holes was considered by Schützhold and Unruh [16], and more extensively by Basak and Majumdar [17, 18], who computed analytically the reflection coefficients in the low frequency limit $\omega A / c^{2} \ll 1$. In particular, the authors of [16] showed that the ergoregion instability in gravity wave analogues is related to the existence of an "energy function" [their Eq. (68)] that is not positive definite inside the ergosphere. In the context of analogues, inertial superradiance based on superfluid ${ }^{3} \mathrm{He}$ has been studied by Jacobson and Volovik [57].

Here we present a quantitative calculation of the efficiency of superradiant amplification for the draining bathtub metric. We work in the frequency representation, and consider an incident plane wave of unit amplitude at infinity, frequency $\omega$ and azimuthal index $m$. Part of this wave will be reflected back by the medium, the reflection coefficient being some (complex) number $R_{\omega m}$. In terms of the wave equation (16), this determines the following boundary condition at infinity:

$$
H \sim R_{\omega m} e^{\mathrm{i} \omega r_{*}}+e^{-\mathrm{i} \omega r_{*}}, r \rightarrow \infty .
$$

At the sonic horizon $\left(r \rightarrow 1, r_{*} \rightarrow-\infty\right)$ the solution behaves like

$$
H \sim T_{\omega m} e^{-\mathrm{i}(\omega-m B) r_{*}}, r \rightarrow 1 .
$$

where $T_{\omega m}$ is the transmission coefficient. An easy way to prove the existence of superresonance is to compute the Wronskian of a solution of equation (16) and of its adjoint at the sonic horizon and at infinity. From the constancy of the Wronskian as a function of the radial coordinate, using the boundary conditions (39) and (40) we find the following "energy conservation" condition:

$$
1-\left|R_{\omega m}\right|^{2}=\left(1-\frac{m B}{\omega}\right)\left|T_{\omega m}\right|^{2} .
$$

Therefore, if $\omega<m B$ the reflection coefficient $\left|R_{\omega m}\right|^{2}>1$, i.e. we have superradiance.

To compute numerically with improved accuracy the reflection coefficient $R_{\omega m}$, we have used a refined condition at the horizon:

$$
H \sim T_{\omega m} e^{-\mathrm{i}(\omega-m B) r_{*}}\left[1+y_{1}(r-1)+\ldots\right],
$$

where the leading-order coefficient is

$$
y_{1}=\frac{\left(1+2 B^{2}\right) m^{2}-2 \omega B m+1}{2[\mathrm{i}(m B-\omega)+1]} .
$$

We also keep higher-order terms to extract the reflection coefficient at infinity. Namely, for the outgoing wave we use an expansion of the form:

$$
H \sim e^{\mathrm{i} \omega r_{*}}\left[1+\frac{z_{1}}{r}+\frac{z_{2}}{r^{2}}+\ldots\right]
$$

where

$$
z_{1}=\mathrm{i} \frac{B+\left(4 m^{2}-1\right)}{8 \omega}, \quad z_{2}=\mathrm{i} z_{1}\left[\frac{B m}{2}+\frac{\left(4 m^{2}-9\right)}{16 \omega}\right],
$$

and for the ingoing wave we use the complex conjugate of Eq. (44). As a check of our code we have reproduced results by Andersson et al. [58] for the superradiant amplification of a scalar field in the vicinities of a Kerr black hole. Our results are in perfect agreement with Fig. 1 of [58]. In particular, we find a maximum amplification coefficient given by $1-\left|R_{\omega m}\right|^{2} \simeq 0.2 \%$ for $l=m=2$ scalar perturbations of a near-extremal extremal Kerr black hole (in early numerical work $\left[19,[56]\right.$ the maximum amplification was found to be $1-\left|R_{\omega m}\right|^{2} \simeq 0.3 \%$ ).

Results of the numerical integrations for the draining bathtub metric are shown in Fig. 2] Panels on the left show the reflection coefficient $\left|R_{\omega 1}\right|^{2}$ for $m=1$, and panels on the right show $\left|R_{\omega 2}\right|^{2}$ for $m=2$, for selected values of the black hole rotation $B$. Panels on top show that, as expected, in the superradiant regime $0<\omega<m B$ the reflection 

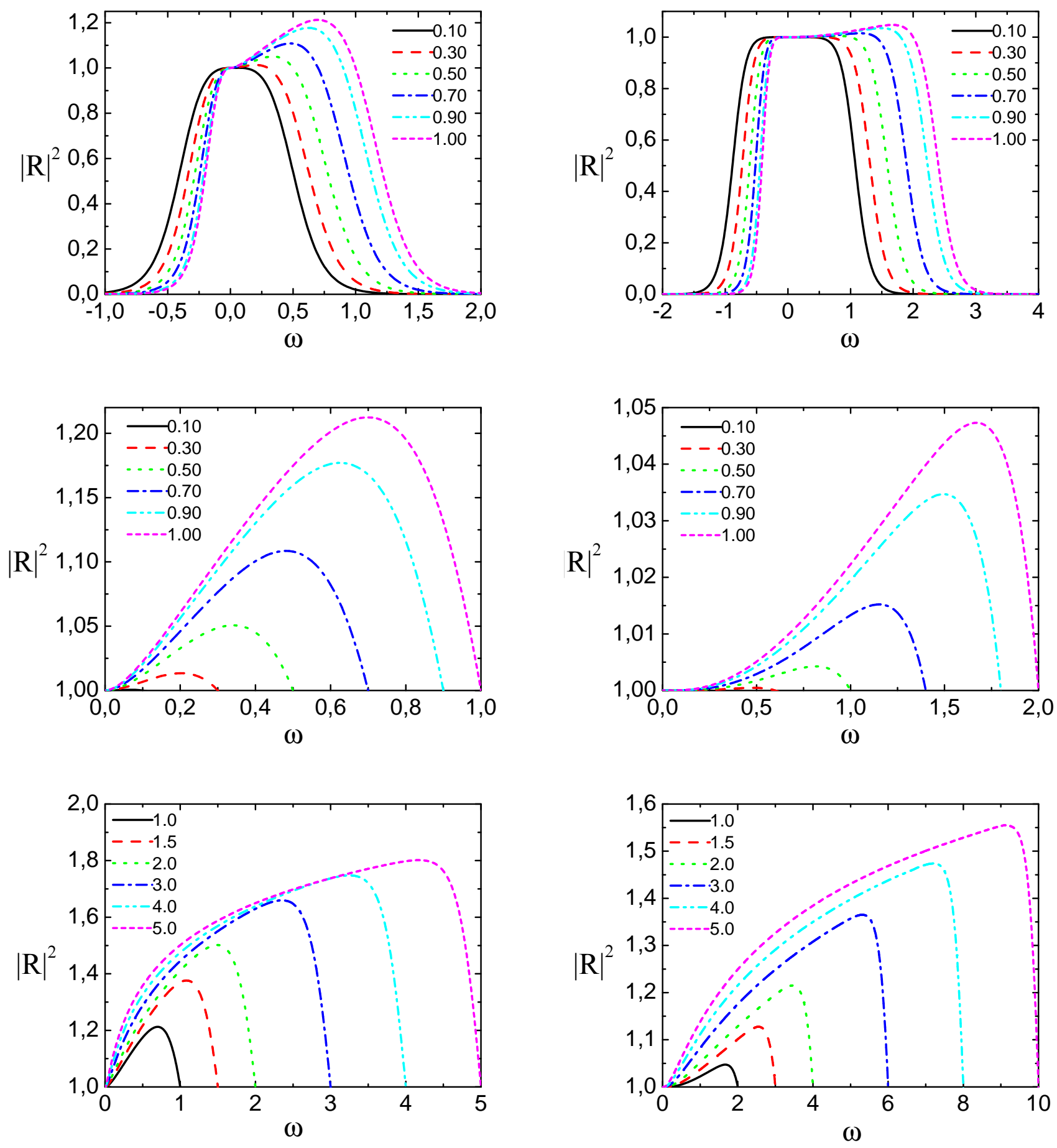

FIG. 2: Reflection coefficient $\left|R_{\omega m}\right|^{2}$ as a function of $\omega$ for $m=1$ (left panels) and $m=2$ (right panels). Each curve corresponds to a different value of $B$, as indicated. The top panels show that the reflection coefficient decays exponentially at the critical frequency for superradiance, $\omega_{S R}=m B$. The middle panels show a close-up view in the superradiant regime for $B<1$ : at $B=1$ the maximum amplification is $21.2 \%(m=1)$ and $4.7 \%(m=2)$. The bottom panels show that superradiant amplification can become much more efficient for values of the rotation parameter $B>1$. 
coefficient $\left|R_{\omega m}\right|^{2} \geq 1$. Furthermore, as one increases $B$ the reflection coefficient increases, and for fixed $B$, the reflection coefficient $\left|R_{\omega m}\right|^{2}$ attains a maximum at $\omega \sim m B$, after which it decays exponentially as a function of $\omega$ outside the superradiant interval. This is very similar to what happens when one deals with massless fields in the vicinities of rotating Kerr black holes 19]. In particular, from the close-up view in the middle panels we see that, for $B=1$, the maximum amplification is $21.2 \%(m=1)$ and $4.7 \%(m=2)$.

As a final remark, and as we have anticipated, an important difference between the acoustic black hole metric and the Kerr metric is that in the present case there is no mathematical upper limit on the black hole's rotational velocity $B$. In the bottom panels we show that, considering values of $B>1$, we can indeed have larger amplification factors for acoustic black holes.

Summarizing: if we are clever enough to build in the lab an acoustic black hole that spins very rapidly, rotational superradiance can be particularly efficient in analogues. This is an important result, considering that the detection of rotational superradiance in the lab is by no means an easy task, as originally predicted by Zel'dovich [50] and confirmed by recent reconsiderations of the problem [49]. Of course, in any real-world experiment the maximum rotational parameter will be limited. At the mathematical level, the equations describing sound propagation (which are written assuming the hydrodynamic approximation) will eventually break down. Physically, if the angular component of the velocity $v^{\theta}$ becomes very large the dispersion relation for the fluid will change, invalidating the assumptions under which we have derived our acoustic metric [16]. To be more concrete, let us consider again the gravity wave analogue described in Section IIB Then we can use a very simple argument to limit the acoustic black hole spin. The derivation of the rotating acoustic black hole metric is based on the assumption that the bottom of the tank should not be too steep, that is, $f^{\prime}(r) \ll 1$. This condition translates into a condition for $B$ :

$$
B \ll\left(\frac{g r^{3}}{2}-A^{2}\right)^{1 / 2}
$$

which must be satisfied for all values of $r$, and in particular at the acoustic black hole horizon $r=r_{H}$ (notice that here, and here only, we have switched back to physical units). Setting $r=r_{H}$ in the previous inequality and using the physical parameters quoted in Section IB] we get the very stringent condition that $B \ll 2.2 \mathrm{~m}^{2} \mathrm{~s}^{-1}$, or (in the dimensionless units we use throughout this paper) $\hat{B} \ll 7.0$. In other words, we can only get values of the rotation parameter larger than $\hat{B} \sim 1$ when the slope of the tank is so large that the assumptions underlying the derivation of the acoustic metric are not valid any more. Of course, this example does not mean that such a constraint applies to every possible experimental realization of the draining bathtub metric. However, it serves as an illustration of the kind of experimental difficulties we may expect to encounter in practice.

The superradiant phenomena we have described are purely classical in nature. However, an interesting suggestion to observe quantum effects in acoustic superradiance was put forward in [18]. To write down our acoustic metric we required the flow to be irrotational and nonviscous. As a natural choice, we could use a fluid which is well known to possess precisely these properties: superfluid HeII. In this case the presence of vortices with quantized angular momenta may lead to a quantized energy flux. The heuristic argument presented in [18] goes as follows. Let us imagine that our black hole is a vortex with a sink at the centre. In the quantum theory of HeII the wavefunction is of the form $\Psi=\exp \left[\mathrm{i} \sum_{j} \phi\left(\vec{r}_{j}\right) \Phi_{\text {ground }}\right]$, where $\vec{r}_{j}$ is the position of the $j$-th particle of HeII. The velocity at any point is given by the gradient of the phase at that point, $\vec{v}=\nabla \phi$, so that (roughly speaking) the velocity potential (3) can be identified with the phase of the wavefunction. This phase will be singular at the sink $r=0$. Continuity of the phase around a circle surrounding the sink requires that the change of the wavefunction satisfies $\Delta \phi=2 \pi B$. For the wavefunction to be single valued, $B$ (that is, the black hole's angular velocity at the horizon) must be the integer multiple of some minimum value $\Delta B$, i.e., $B=n \Delta B$. Then the angular momentum of the acoustic black hole would be forced to change in integer multiples of $\Delta B$. Correspondingly, the spectrum of the reflection coefficients may be given by equally-spaced peaks with different strengths. This discrete amplification could enhance chances of observing superradiance in acoustic black holes, and rule out (or provide empirical support to) some of the many competing heuristic approaches to black hole quantization.

\section{F. Superradiant instabilities: the acoustic black hole bomb}

The very existence of an ergoregion in the acoustic black hole metric allows immediately for the possibility to make the system unstable. Suppose we enclose the system by a reflecting mirror at constant radius $r_{0}$. Now, throw in a wave having frequency $\omega=\omega_{R}+\mathrm{i} \omega_{I}$ such that $\omega_{R}<m \Omega$ : the wave will be amplified at the expense of the black hole's rotational energy and travel back to the mirror. There it will be reflected and move again towards the black hole, this time with increased amplitude. Through repeated reflections, the waves' amplitude will grow exponentially 

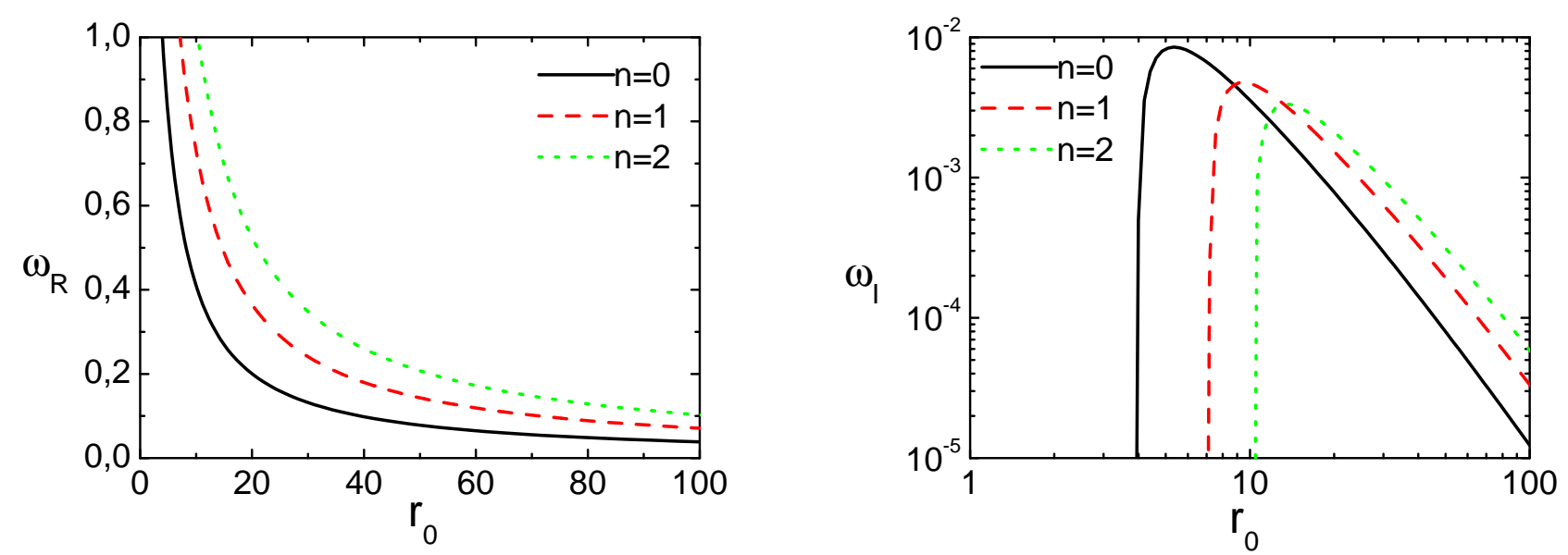

FIG. 3: Real part (left) and imaginary part (right) of the fundamental BQN frequency and the first two overtones as a function of mirror location $r_{0}$. Both panels refer to $m=1$ and $B=1$ (but the $B$-dependence of the real part of the BQN frequency is very weak). With excellent accuracy $\omega_{I}$ crosses zero (and the instability abruptly shuts off) at the critical radius predicted by Eq. (48), as can be verified taking a glance at the first row of Table

with time. This "black hole bomb" was first proposed by Press and Teukolsky [56], and recently it has been studied in detail by some of us 20] in the context of the Kerr metric. The detailed analysis performed in 20] showed that the black hole bomb can be characterized by a set of complex resonant frequencies, the Boxed QuasiNormal Modes (BQNMs). The real part of a BQNM is, not surprisingly, proportional to $1 / r_{0}$, where $r_{0}$ is the mirror radius: this is essentially the condition for the existence of standing waves in the region enclosed by the mirror. The imaginary part of the BQNMs is proportional to $\left(\omega_{R}-m \Omega\right)$ : the system can only become unstable if it is in the superradiant regime. Combined with the standing wave condition this implies that the mirror should be placed at some radius $r_{0} \gtrsim 1 / m \Omega$ in order for the system to be unstable. As rotational energy is extracted from the system the black hole will spin down. For any given $r_{0}$ the instability will eventually shut off, as the condition $r_{0} \gtrsim 1 / m \Omega$ will no longer be satisfied.

An additional reason to study the system "acoustic black hole+mirror" is related to the problem of boundary conditions at infinity for QNMs (cf. Section IC). Whatever analogue model we consider, no physical apparatus will ever extend out to infinity. We are left with two possibilities: we can either use some absorbing device to simulate spatial infinity, or we can impose alternative boundary conditions on the system. A natural choice is to have a reflecting mirror surrounding the apparatus, so that we are effectively building an acoustic black hole bomb.

In the following we will assume that we are in the presence of a perfectly reflecting mirror. Correspondingly, we shall impose the boundary condition $H=0$ at $r=r_{0}$. At the horizon we demand, as usual, the presence of purely ingoing waves (waves headed towards the horizon), i.e. $H \sim e^{-\mathrm{i}(\omega-B m) r_{*}}$. We are again in presence of an eigenvalue problem. However, since the boundary conditions have changed we shall not refer to the characteristic frequencies as QN frequencies, but rather as Boxed QuasiNormal frequencies (BQN frequencies). The reader is referred to 20], where this terminology was first introduced. Using a direct integration of the wave equation (see Section IIE for details) it is quite easy to compute the BQN frequencies. We simply integrate Eq. (16) outwards from the horizon, where we impose the condition (42), to the mirror location $r_{0}$. The (complex) BQNM frequencies are those frequencies for which

$$
H\left(\omega_{B Q N M}, r_{0}\right)=0 .
$$

Some results for the fundamental BQNM and the first two overtones are shown in Fig. 3 There we fixed the black hole rotation parameter $B=1$, and $m=1$, as a typical case.

From the left panel we see that the real part of the BQNM frequency for the $n$-th overtone (where we use the convention that $n=0$ corresponds to the fundamental mode) scales as $1 / r_{0}$, as anticipated. The proportionality constant can be obtained by analytical arguments similar to those in [20]. The result is

$$
\omega_{R}=\frac{j_{m, n}}{r_{0}},
$$

where the $j_{m, n}$ 's are zeros of the Bessel function of integer order $m$ (recall that for a Kerr black hole bomb one gets $\omega_{R}=j_{l+1 / 2, n} / r_{0}$ instead, cf. [20]). The numerical values of the $j_{m, n}$ 's can be found, e.g., in [59]. For reference, we list 
TABLE II: Zeros of the Bessel functions $j_{m, n}$ for the first few values of $m$. The real part of the BQNM frequency is well approximated by Eq. (47).

\begin{tabular}{c|c|c|c}
\hline \hline$m$ & $j_{m, 0}$ & $j_{m, 1}$ & $j_{m, 2}$ \\
\hline \hline 1 & 3.83171 & 7.01559 & 10.17347 \\
2 & 5.13562 & 8.41724 & 11.61984 \\
3 & 6.38016 & 9.76102 & 13.01520 \\
4 & 7.58834 & 11.06471 & 14.37254 \\
5 & 8.77148 & 12.33860 & 15.70017 \\
\hline \hline
\end{tabular}
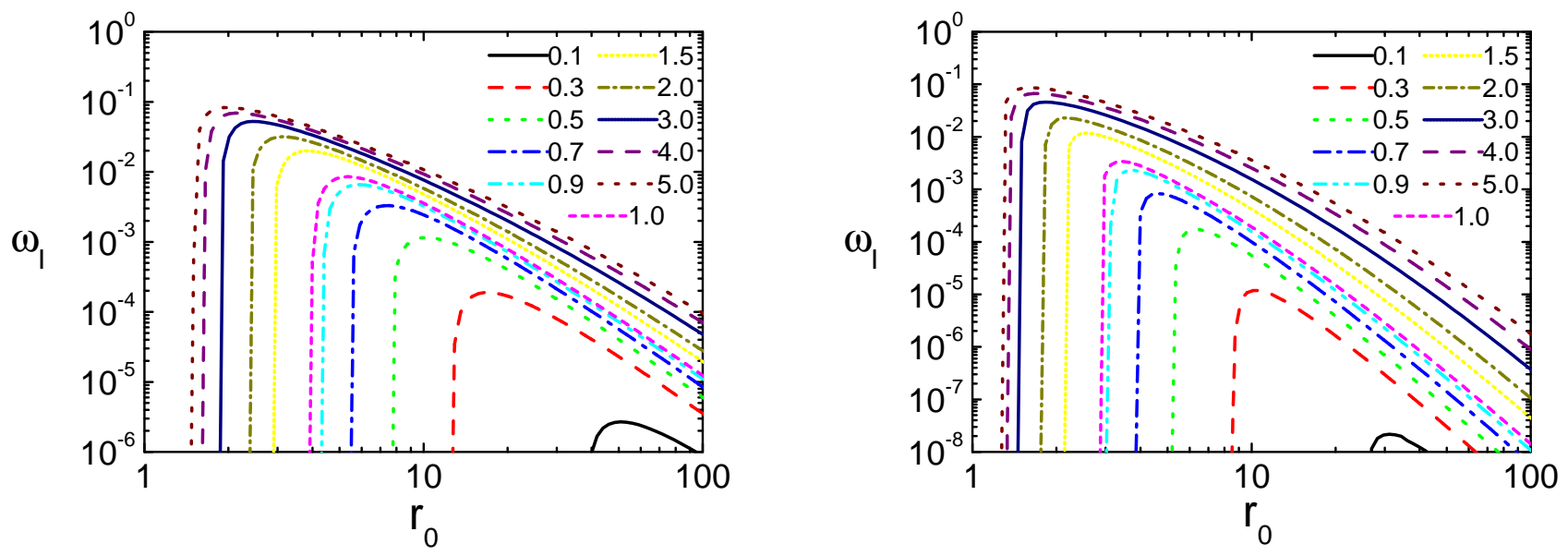

FIG. 4: Growth timescales for BQNMs, as a function of mirror location $r_{0}$, for different values of $B$. The left panel refers to $m=1$, the right panel to $m=2$. Notice the different scales for the $\omega_{I}$ axis.

the first few values in Table III Our numerical calculations are in excellent agreement with the predictions of formula (47). This is particularly true for small values of $B$, but the $B$-dependence of the real part of the BQN frequencies is very weak anyway, as it is for the Kerr metric [20].

From the right panel we see the behavior predicted by the qualitative arguments at the beginning of this Section: for any BQNM, the instability is more and more efficient as the mirror radius $r_{0}$ becomes smaller, until eventually the mirror radius becomes small enough that the instability shuts off.

A few remarks are in order: (i) The real part of the BQN frequency (slowly) increases with overtone number $n$. (ii) Higher overtones become stable at larger distances, but they also attain a smaller maximum growing rate. (iii) With excellent accuracy, the instability switches off at the critical radius predicted by the analytical formula (47) supplemented by the superradiance condition (38), that is:

$$
r_{0, c} \simeq \frac{j_{m, n}}{m B} .
$$

Since $j_{m, n}$ grows linearly with $m$ (in particular, this is asymptotically true for high $m$ 's [59]) the critical radius is almost $m$-independent. However, the instability is not so efficient for higher $m$ as it is for small $m$, at least when $B \lesssim 1$. This is apparent in Fig. 目 where we compare growth timescales with $m=1$ (left) and $m=2$ (right) for acoustic black holes rotating at different rates. For example, the maximum growth rate for $B=0.3$ is $\sim 10^{-4}$ for $m=1$, and $\sim 10^{-5}$ for $m=2$. The growth rate for $m=2$ becomes roughly comparable to the growth rate for $m=1$ when $B>1$ : look for example at the curves corresponding to $B=5.0$. However, other physical considerations may forbid the construction of acoustic black holes rotating at such rates (see the arguments at the end of Section IIE).

To get an idea of the orders of magnitude involved, let us consider again the "typical" parameters for the gravity wave analogue we introduced in Section $\amalg B$ Let us pick $m=1$ and an acoustic black hole rotation parameter $\hat{B}=1$, which - according to the arguments in Section [IE - is close to the maximum rotation rate we may hope to achieve (recall hats distinguish dimensionless quantities from quantities in physical units). To take advantage of the process the mirror should be located close to the maximum of $\omega_{I}$, but not quite at the maximum. For example, if we place the mirror at $\hat{r}_{0} \simeq 10$ (cf. the right panel of Fig. 3) we get $\hat{\omega}_{I} \simeq 4 \cdot 10^{-3}$ (in this case the maximum growth rate 
would be $\hat{\omega}_{I}=8.5 \cdot 10^{-3}$ ). This corresponds to a growth time $\tau \simeq 800 \mathrm{~s} \simeq 13$ minutes (at the maximum we would get $\tau \simeq 6$ minutes). Roughly speaking, this means that the amplitude will double every 13 minutes or so, and will be amplified by orders of magnitude on timescales of the order of a few hours. To reduce the typical timescale to seconds, one just has to ajust the horizon radius and wave velocity. For example, working with $r_{H} \sim 0.1 \mathrm{~m}$ and $c \sim 10 \mathrm{~m} \mathrm{~s}^{-1}$, we would get a typical timescale of about 2 seconds. This looks like a perfectly reasonable timescale to observe an acoustic black hole bomb in the lab.

When the instability sets in the acoustic black hole loses energy and angular momentum $(\Delta E \sim B \Delta J)$. The critical radius $r_{0, c}$ increases with decreasing $B$. To estimate the efficiency we can use a simple argument. We can take advantage of the mechanism by picking some large $B$ and $r_{0} \simeq 2 r_{0, \text { max }}$, where $r_{0, \text { max }}$ is the radius of maximum growth timescale at the given $B$. As the hole emits angular momentum $B$ will decrease. For any given mirror location $r_{0}$, the bomb eventually switches off when condition (48) is satisfied. From the difference in initial and final angular momentum $\Delta B$ we can infer the extracted energy $\Delta E$. For more details we refer to [20].

As a final remark we want to stress that, when we talk about surrounding the acoustic black hole by a reflecting mirror, what we have in mind is a generic type of mirror. For instance, in the shallow basin gravity wave analogue, the mirror could be a circular rubber band of radius $r_{0}$ reflecting gravity waves. A possible alternative to implement a mirror could involve, for example, variations in the dispersion relation for sound waves. In particular, Schützhold and Unruh [16] suggested that a simple change of the height $h_{\infty}$ of the basin would imply a change of the gravity wave speed, hence of the effective refractive index. The changed refractive index may be used to trap the waves, which can then be amplified via superradiant scattering until non-linear effects dominate.

\section{THE CANONICAL NON-ROTATING ACOUSTIC BLACK HOLE}

In the previous Section we considered the metric describing a rotating $(2+1)$-dimensional acoustic black hole. Rotation implies the presence of an ergosphere, allowing us to study in the lab some of the most interesting phenomena concerning black hole physics (eg. superradiance and the related rotational instabilities). However, the non-rotating limit of the acoustic metric we considered is not the "natural" metric describing a non-rotating acoustic black hole. In this Section we are going to consider another class of non-rotating, $(3+1)$-dimensional acoustic metrics. These metrics are associated to the most general spherically symmetric flow of an incompressible fluid: in this sense they represent "canonical" metrics for a non-rotating acoustic black hole.

\section{A. Formalism and basic equations}

It is simple to show that the acoustic metric corresponding to the most general spherically symmetric flow of an incompressible fluid is given by [7]

$$
d s^{2}=-c^{2}\left(1-\frac{r_{0}^{4}}{r^{4}}\right) d t^{2}+\left(1-\frac{r_{0}^{4}}{r^{4}}\right)^{-1} d r^{2}+r^{2}\left(d \theta^{2}+\sin \theta^{2} d \phi^{2}\right) .
$$

This metric does not correspond to any of the geometries typically considered in general relativity, but it describes (in the sense specified above) a "canonical" acoustic black hole. The propagation of small disturbances (sound waves) is again described by the massless Klein-Gordon equation $\nabla_{\mu} \nabla^{\mu} \Psi=0$ in this background. We can separate variables by the substitution

$$
\Psi(t, r, \phi)=\frac{\Phi(\omega, r)}{r} e^{-\mathrm{i} \omega t} Y_{l m}(\theta),
$$

where $Y_{l m}(\theta)$ are the usual spherical harmonics. This yields the wave equation

$$
\Phi_{, r_{*} r_{*}}+\left(\frac{\omega^{2}}{c^{2}}-V\right) \Phi=0
$$

where

$$
V=\left(1-\frac{r_{0}^{4}}{r^{4}}\right)\left[\frac{l(l+1)}{r^{2}}+\frac{4 r_{0}^{4}}{r^{6}}\right]
$$

and the tortoise coordinate $r_{*}$ is defined, as usual, by $d r / d r_{*}=\left(1-r_{0}^{4} / r^{4}\right)$. Since this is a spherical symmetric problem, the azimuthal number $m$ does not play any role, only the angular momentum $l$ is important here. In the 
TABLE III: The fundamental $(n=0)$ QN frequencies for the non-rotating canonical acoustic black hole, using three WKB computational schemes. $\omega_{Q N}^{(1)}$ is the result for the QN frequency using only the lowest approximation [25], $\omega_{Q N}^{(3)}$ is the value obtained using 3rd order improvements [26], and finally $\omega_{Q N}^{(6)}$ was computed using 6th order corrections [28].

\begin{tabular}{c|c|c|c}
\hline \hline$l$ & $\omega_{Q N}^{(1)}$ & $\omega_{Q N}^{(3)}$ & $\omega_{Q N}^{(6)}$ \\
\hline \hline 0 & $1.13-0.73 \mathrm{i}$ & $0.19-1.11 \mathrm{i}$ & $0.06+0.87 \mathrm{i}$ \\
1 & $1.37-0.68 \mathrm{i}$ & $0.53-0.71 \mathrm{i}$ & $1.09-0.39 \mathrm{i}$ \\
2 & $1.82-0.64 \mathrm{i}$ & $1.41-0.61 \mathrm{i}$ & $1.41-0.70 \mathrm{i}$ \\
3 & $2.36-0.62 \mathrm{i}$ & $2.10-0.62 \mathrm{i}$ & $2.12-0.62 \mathrm{i}$ \\
4 & $2.94-0.62 \mathrm{i}$ & $2.75-0.62 \mathrm{i}$ & $2.75-0.62 \mathrm{i}$ \\
\hline \hline
\end{tabular}

next Section we summarize our results for QNMs and wave tails of the canonical acoustic black hole. When presenting our numerical results we will choose units such that $c=r_{0}=1$. This is of course equivalent to a simple rescaling of the radial variable $\left(\hat{r}=r / r_{0}\right)$ and of the frequency $(\hat{\omega}=\omega / c)$, but we will omit hats in the following.

\section{B. Quasinormal modes}

A 6th order WKB analysis 28] reveals an unstable QNM for $l=0$. However, it is possible to prove stability of the canonical black hole since the potential is positive-definite. We therefore computed the QN frequencies using first the lowest approximation [25], then 3rd order corrections [26] and finally 6th order corrections. The results are presented in Table III The QN frequencies for $l=0$ and $l=1$ seem to be the problem here. For any other $l$ the value quickly converges as we increase the correction order of the WKB method. Notice for example that the $l=0$ mode suffers a variation of almost an order of magnitude as we go from the lowest approximation to the 3rd order correction scheme, and gets unstable for the scheme using 6th order corrections. The reason for this failure is most likely related to the breakdown of the basic WKB assumptions, namely that the ratio of the derivatives of the potential to the potential itself is small. Indeed a close inspection shows that as $l$ increases these ratios tend to decrease. This means that the WKB method is more reliable for higher $l$ : this was first observed in the early works on the subject [25, 26]. For $l=0$ and $l=1$ the lowest WKB approximation gives the most reliable results. This is confirmed by recent numerical work [36.

In the limit of large $l$ one finds

$$
\omega \sim \frac{l \sqrt{2}}{3^{3 / 4}}-\mathrm{i} \frac{\sqrt{2}(1+2 n)}{3^{3 / 4}},
$$

a result which agrees very well with our WKB data already for $l=3$.

The calculation of highly damped QNMs proceeds along the same lines sketched for the $(2+1)$-dimensional acoustic black hole. In this case the index $j=3 / 5$, which implies that

$$
4 \pi \omega=\log \frac{3-\sqrt{5}}{2}-\mathrm{i}(2 n+1) \pi .
$$

Once again asymptotic QN frequencies are not given by the logarithm of an integer, as required in Hod's construction 12. As we remarked earlier this does not necessarily imply that the conjecture is wrong, since a thermodynamical interpretation of the black hole area is possible only when the dynamics of the system are described by the Einstein equations.

\section{Late-time tails}

The analysis of late-time tails proceeds as in the case of the $(2+1)$-dimensional acoustic black hole. We can easily show that asymptotically the potential behaves as

$$
V \sim \frac{l(l+1)}{r_{*}^{2}}+\frac{12-5 l(l+1)}{3 r_{*}^{6}}, r \rightarrow \infty .
$$


Following the previous analysis we now find $\alpha=6$, and since $l$ is an integer the power-law falloff is of the form

$$
\Phi \sim t^{-(2 l+6)} .
$$

Thus any perturbation eventually dies off as $t^{-(2 l+6)}$, much more quickly (for $m=l$ ) than in the $(2+1)$-dimensional acoustic black hole background.

\section{CONCLUSIONS}

In this paper we have considered two acoustic black hole metrics: the $(2+1)$-dimensional "draining bathtub" metric, and the $(3+1)$-dimensional canonical non-rotating acoustic black hole. We have studied QNMs and late-time tails in both metrics, and superradiance in the "draining bathtub" metric.

More specifically, we numerically computed slowly damped QNMs of the draining bathtub metric using a thirdorder WKB approach for all values of $m \neq 0$. We analytically evaluated QN frequencies in the large- $m$ limit [Eq. [23)] and set upper limits on frequencies of unstable modes (Appendix $\mathrm{A}$. We showed that highly damped modes do not tend to any simple limit. At late times, power-law tails decay as $t^{-(2 m+1)}$. This behavior is typical of any odddimensional spacetime: independently of the presence of a black hole, if $D$ is odd the power-law falloff is proportional to $t^{-(2 l+D-2)}$ 47] [the $(2+1)$-dimensional case, $D=3$, is special: the azimuthal and angular numbers are the same, $l=m$, since there is only one angular coordinate]. The draining bathtub metric, possessing an ergoregion, can superradiantly amplify waves. We computed reflection coefficients for this superradiant scattering by numerical integration. When the (dimensionless) acoustic black hole rotation $B=1$, the maximum amplification is $21.2 \%$ for $m=1$ and $4.7 \%$ for $m=2$. Enclosing the acoustic black hole by a reflecting mirror we can destabilize the system, making an initial perturbation grow exponentially with time: we have an acoustic black hole bomb [19], or "dumb hole bomb". We computed analytically and numerically the frequencies and growing timescales for this instability. An interesting feature of acoustic geometries is that the acoustic black hole spin can be varied independently of the black hole mass. Therefore, at variance with the Kerr metric, the spin can be made (at least in principle) arbitrarily large, and rotational superradiance in acoustic black holes can be very efficient. Gravity waves in shallow water [16] provide a concrete example of an experimental setup for studying classical physics in a draining bathtub metric [and of experimental difficulties in increasing arbitrarily the rotation rate: see the discussion leading to Eq. (45)]. In this case, and for a typical choice of parameters, growth times for the black hole bomb instability would be of the order of minutes: this appears to be well within the range of experimental possibilities. Finally one can speculate, following [18], that superfluid HeII could be used to observe some quantum effects, such as a discrete superradiant amplification.

For the canonical acoustic black hole we computed slowly-damped QNMs using first, third and sixth order WKB. For $l<2$ the WKB method does not seem to converge, and even yields an unstable frequency at 6 th order when $l=0$. This is only due to bad convergence properties of the WKB technique, since the canonical acoustic black hole is stable. In the high-damping limit QNMs are given by $4 \pi \omega=\log [(3-\sqrt{5}) / 2]-\mathrm{i}(2 n+1) \pi$, so they are not proportional to the logarithm of an integer, as required by recent conjectures. This is not surprising since there are no Einstein equations for acoustic metrics, i.e., the acoustic metric evolution is not governed by Einstein's equations. Therefore, acoustic black holes can teach us a lot about quantum gravity [7, 10], but cannot shed any light on its possible connection with highly damped quasinormal modes.

The late time falloff in the canonical acoustic black hole metric is proportional to $t^{-(2 l+6)}$, to be compared with the $t^{-(2 l+3)}$ decay of 4 -dimensional Schwarzschild black holes and with the $t^{-(2 l+3 D-8)}$ decay of even-dimensional spherically symmetric black holes with $D>4$ [47].

\section{Acknowledgements}

We are grateful to Carlos Barceló and Ted Jacobson for useful discussions. This work was partially funded by Fundação para a Ciência e Tecnologia (FCT) - Portugal through project CERN/FNU/43797/2001. V.C. acknowledges financial support from FCT through grant SFRH/BPD/2003. This work was supported in part by the National Science Foundation under grant PHY 03-53180.

\section{APPENDIX A: UPPER BOUNDS FOR QUASINORMAL FREQUENCIES OF UNSTABLE MODES}

To derive some bounds on the magnitude of QN frequencies of possible unstable QNMs we shall follow Detweiler and Ipser [60]. Let us begin with the Klein-Gordon equation for the evolution of the field, $\nabla_{\mu} \nabla^{\mu} \Psi=0$. Using the 
metric (14) and performing a mode decomposition $\Psi(t, r, \phi)=R(r) e^{\mathrm{i}(m \phi-\omega t)}$, this can be written as

$$
\frac{r}{f c^{2}} \omega^{2} R+\frac{d}{d r}\left[r f R^{\prime}\right]-\frac{2 B m \omega}{c^{2} f r} R-\left(\frac{1}{r}-\frac{B^{2}}{c^{2} r^{3} f}\right) m^{2} R=0,
$$

where we have defined $f \equiv \Delta^{-1}=1-A^{2} / c^{2} r^{2}$.

Multiply by $R^{*}$ and integrate from the horizon to spatial infinity. The result is

$$
\int_{r_{H}}^{\infty} d r\left[\frac{r}{f c^{2}} \omega^{2}|R|^{2}-r f\left|R^{\prime}\right|^{2}-\frac{2 B m \omega}{c^{2} f r}|R|^{2}-\left(\frac{1}{r}-\frac{B^{2}}{c^{2} r^{3} f}\right) m^{2}|R|^{2}\right]=0,
$$

where we have used an integration by parts and discarded the surface integrals. In fact, for unstable modes (and for these only) the boundary conditions guarantee that an unstable mode vanishes exponentially as $r \rightarrow r_{H}$ or $r \rightarrow \infty$. The imaginary part of this equation yields

$$
\int_{r_{H}}^{\infty} d r\left(r^{2} \omega_{R}-B m\right) \frac{|R|^{2}}{f r c^{2}}=0
$$

where we used $\omega=\omega_{R}+\mathrm{i} \omega_{I}$. Therefore $\omega_{R}$ and $B m$ must have the same sign for unstable modes. Furthermore, since $|R|^{2} / f r c^{2}$ is always positive, the quantity $\left(r^{2} \omega_{R}-B m\right)$ must be negative somewhere for the integral to vanish. Since $r^{2} \omega_{R}$ increases with $r$, if this quantity is somewhere negative, then it certainly is negative at the horizon. In this way we get an upper bound for $\omega_{R}$ :

$$
\omega_{R}<\frac{B m}{r_{H}^{2}} .
$$

To get a similar bound on $\omega_{I}$ consider now the real part of Eq. (A2):

$$
\int_{r_{H}}^{\infty} d r\left[\omega_{I}^{2}-\omega_{R}^{2}+\frac{2 B m \omega_{R}}{r^{2}}+\frac{c^{2} f m^{2}}{r^{2}}-\frac{B^{2} m^{2}}{r^{4}}+f^{2} c^{2} \frac{\left|R^{\prime}\right|^{2}}{|R|^{2}}\right] \frac{r|R|^{2}}{f c^{2}}=0 .
$$

The positive part of the integrand is (assuming without loss of generality that $B m \omega_{R}>0$ )

$$
\omega_{I}^{2}+\frac{2 B m \omega_{R}}{r^{2}}+\frac{c^{2} f m^{2}}{r^{2}}+f^{2} c^{2} \frac{\left|R^{\prime}\right|^{2}}{|R|^{2}}>\omega_{I}^{2} .
$$

The negative part is maximized at the horizon:

$$
\omega_{R}^{2}+\frac{B^{2} m^{2}}{r^{4}}<\omega_{R}^{2}+\frac{B^{2} m^{2}}{r_{H}^{4}} .
$$

A necessary condition for the integral to vanish is that the integrand be negative at the horizon, and this implies

$$
\omega_{I}^{2}<\omega_{R}^{2}+\frac{B^{2} m^{2}}{r_{H}^{4}}
$$

Using the bound A4 for $\omega_{R}$ we finally get an upper bound for $\omega_{I}$ :

$$
\omega_{I}^{2}<\frac{2 B^{2} m^{2}}{r_{H}^{4}}
$$

Notice that the upper bound for $\omega_{R}$ ensures we are in the superradiant (or better, superresonant) regime. This seems to be a general feature, since it has been shown to hold in several black hole spacetimes. If the instability sets in at all, the system behaves as a black hole bomb.

[1] S. W. Hawking, Nature 248, 30 (1974); S. W. Hawking, Commun. Math. Phys. 43, 199 (1975).

[2] M. Visser, Phys. Rev. Lett. 80, 3436 (1998).

[3] M. Visser, Int. J. Mod. Phys. D 12, 649 (2003). 
[4] S. B. Giddings, S. Thomas, Phys. Rev. D 65, 056010 (2002).

[5] W. G. Unruh, Phys. Rev. Lett. 46, 1351 (1981).

[6] M. Novello, M. Visser and G. Volovik (editors), Artificial black holes (World Scientific, Singapore, 2002).

[7] M. Visser, Class. Quantum Grav. 15, 1767 (1998).

[8] C. Barceló, S. Liberati and M. Visser, Int. J. Mod. Phys. A 18, 3735 (2003).

[9] R. Schutzhold and W. G. Unruh, quant-ph/0408145

[10] S. Corley and T. Jacobson, Phys. Rev. D 59, 124011 (1999).

[11] S. Detweiler, Phys. Rev. D 22, 2323 (1980); T. J. Zouros and D. M. Eardley, Annals Phys. 118, 139 (1979); T. Damour, N. Deruelle and R. Ruffini, Lett. Nuovo Cim. 15, 257 (1976).

[12] S. Hod, Phys. Rev. Lett. 81, 4293 (1998).

[13] K. D. Kokkotas and B. G. Schmidt, Living Rev. Rel. 2, 2 (1999); H.-P. Nollert, Class. Quantum Grav. 16, R159 (1999).

[14] F. Echeverria, Phys. Rev. D 40, 3194 (1989); L. S. Finn, Phys. Rev. D 46, 5236 (1992).

[15] R. H. Price, Phys. Rev. D5, 2419 (1972).

[16] R. Schützhold, W. G. Unruh, Phys. Rev. D 66, 044019 (2002).

[17] S. Basak and P. Majumdar, Class. Quant. Grav. 20, 2929 (2003).

[18] S. Basak and P. Majumdar, Class. Quant. Grav. 20, 3907 (2003).

[19] W. Press and S. Teukolsky, Astrophys. J. 193, 443 (1974).

[20] V. Cardoso, O. J. C. Dias, J. P.S. Lemos and S. Yoshida, Phys. Rev. D70, 044039 (2004); 70, 044039(E)(2004).

[21] For a possible astrophysical relevance of the black hole bomb mechanism, see M. H. P. M. Putten, Science 284, 115 (1999); A. Aguirre, Astrophys. J. 529, L9 (2000).

[22] P. Painlevé, C. R. Hebd. Seances Acad. Sci. 173, 677 (1921); A. Gullstrand, Ark. Mat. Astron. Fys. 16, 1 (1922); G. Lemaitre, Ann. Soc. Sci. Bruxelles, Ser. 1 53, 51 (1933).

[23] S. Chandrasekhar, Hydrodynamic and Hydromagnetic Stability (Dover Publications, New York, 1981).

[24] P. G. Drazin and W. H. Reid, Hydrodynamic Stability (Cambridge University Press, 2004).

[25] B. F. Schutz and C. M. Will, Astrophys. Journal 291, L33 (1985);

[26] C. M. Will and S. Iyer, Phys. Rev. D 35, 3621 (1987); S. Iyer, Phys. Rev. D 35, 3632 (1987); E. Seidel and Sai Iyer, Phys.Rev. D 41, 374 (1990).

[27] K. D. Kokkotas, Class. Quant. Grav. 8, 2217 (1991).

[28] R. A. Konoplya, Phys. Rev. D 68, 024018 (2003).

[29] V. Ferrari and B. Mashhoon, Phys. Rev. D30, 295 (1984).

[30] O. Dreyer, B. Kelly, B. Krishnan, L. S. Finn, D. Garrison and R. Lopez-Aleman, Class. Quantum Grav. 21, 787 (2004).

[31] V. Cardoso, J. P. S. Lemos and S. Yoshida, gr-qc/0410107

[32] O. Dreyer, Phys. Rev. Lett. 90, 081301 (2003).

[33] L. Motl, Adv. Theor. Math. Phys. 6, 1135 (2003).

[34] L. Motl and A. Neitzke, Adv. Theor. Math. Phys. 7, 2 (2003).

[35] H.-P. Nollert, Phys. Rev. D 47, 5253 (1993); E. Berti and K. D. Kokkotas, Phys. Rev. D 68, 044027 (2003).

[36] V. Cardoso, J. P. S. Lemos and S. Yoshida Phys. Rev. D 69, 044004 (2004).

[37] N. Andersson and C. J. Howls, Class. Quantum Grav. 21, 1623 (2004).

[38] V. Cardoso, J. Natário and R. Schiappa, J. Math. Phys. (in press); hep-th/0403132

[39] T. Tamaki and H. Nomura, Phys. Rev. D 70, 044041 (2004); J. Kettner, G. Kunstatter and A.J.M. Medved; gr-qc/0408042 S. Chen and J. Jing, gr-qc/0409013

[40] V. Cardoso, R. Konoplya and J. P. S. Lemos, Phys. Rev. D 68, 044024 (2003); S. Yoshida and T. Futamase, Phys. Rev. D 69, 064025 (2004). R. A. Konoplya and A. Zhidenko, JHEP 0406, 037 (2004).

[41] We thank Andrew Neitzke for sharing with us a related unpublished calculation for the Kerr metric.

[42] T. Jacobson, Phys. Rev. Lett. 75, 1260 (1995).

[43] C. Barceló, S. Liberati, S. Sonego and M. Visser, gr-qc/0408022

[44] C. Gundlach, R. H. Price and J. Pullin, Phys. Rev. D49, 883 (1994); C. Gundlach, R. H. Price and J. Pullin, Phys. Rev. D49, 890 (1994); S. Hod, Phys. Rev. D58, 104022 (1998); L. Barack and A. Ori, Phys. Rev. Lett. 82, 4388 (1999); N. Andersson and K. Glampedakis, Phys. Rev. Lett. 84, 4537 (2000); H. Koyama and A. Tomimatsu, Phys. Rev. D64, 044014 (2001); S. Hod, Class. Quant. Grav. 18, 1311 (2001).

[45] E. S. C. Ching, P. T. Leung, W. M. Suen and K. Young, Phys. Rev. Lett. 74, 2414 (1995).

[46] E. S. C. Ching, P. T. Leung, W. M. Suen and K. Young, Phys. Rev. D52, 2118 (1995).

[47] V. Cardoso, S. Yoshida, O. J. C. Dias and J. P.S. Lemos, Phys. Rev. D 68, 061503 (2003).

[48] V. L. Ginzburg and I. M. Frank, Dokl. Akad. Nauk SSSR 56, 583 (1947); for a recent review cf. V. L. Ginzburg, in Progress in Optics XXXII, edited by E. Wolf (Elsevier, Amsterdam, 1993).

[49] J. D. Bekenstein and M. Schiffer, Phys. Rev. D 58, 064014 (1998).

[50] Ya. B. Zel'dovich, JETP Lett. 14, 180 (1971); Sov. Phys. JETP 35, 1085 (1972).

[51] C. W. Misner, Phys. Rev. Lett. 28, 994 (1972); A. A. Starobinsky, Sov. Phys. JETP 37, 28 (1973); A. A. Starobinsky and S. M. Churilov, Sov. Phys. JETP 38, 1 (1973); W. Unruh, Phys. Rev. D 10, 3194 (1974); W. H. Press and S. A. Teukolsky, Astrophys. Journal 185, 649 (1973).

[52] J. D. Bekenstein, Phys. Rev. D 7, 949 (1973).

[53] R. Penrose, Nuovo Cimento 1, 252 (1969).

[54] J. L. Friedman, Commun. Math. Phys. 63, 243 (1978). 
[55] N. Comins and B. F. Schutz, Proc. R. Soc. Lond. A 364, 211 (1978).

[56] W. H. Press and S. A. Teukolsky, Nature 238, 211 (1972).

[57] T. Jacobson and G. E. Volovik, Phys. Rev. D 58, 064021 (1998).

[58] N. Andersson, P. Laguna and P. Papadopoulos, Phys. Rev. D 58, 087503 (1998).

[59] M. Abramowitz and A. Stegun, Handbook of mathematical functions (Dover Publications, New York, 1970).

[60] S. L. Detweiler and J. R. Ipser, Astrophys. Journal 185, 675 (1973). 